\title{
Packet Loss Analysis of Synchronous Buffer-less Optical Switch with Shared Limited Range Wavelength Converters
}

\author{
Carla Raffaelli, Michele Savi \\ D.E.I.S \\ University of Bologna \\ Bologna, Italy. \\ Email:\{craffaelli,msavi\}@deis.unibo.it
}

\author{
Nail Akar, Ezhan Karasan \\ Electrical and Electronics Engineering \\ Bilkent University \\ Ankara, Turkey. \\ Email:\{akar,ezhan\}@ee.bilkent.edu.tr
}

\begin{abstract}
Application of synchronous optical switches in Optical Packet/Burst switched networks is considered. The shared per node architectural concept, where wavelength converters are shared among all input and output channels, is applied for contention resolution in the wavelength domain. A semi-analytical traffic model suitable to represent the different contributions to packet loss is proposed and validated. Full and limited range wavelength conversion capabilities are considered, and loss results obtained to support switch design. An approximated fully analytical approach for the limited range case is also described and comparison with simulation results is presented to assess the capability to capture the main aspects of packet loss behavior.

Keywords: Optical Packet Switching, Optical Burst Switching, Limited range tunable wavelength converters, performance modeling
\end{abstract}

\section{INTRODUCTION}

Optical packet switched networks have been proposed as a solution for core networks since they are able to exploit the enormous bandwidth offered by optical fibers in efficient way. The optical burst switched solution differs as regards control signaling procedure but has practically the same approach as far as the data plane is concerned. In fact these technologies both exploit statistical multiplexing in the optical layer, to provide finer granularity than wavelength routed counterpart [1], [2]. Photonic packet switching nodes [1], [3] are needed to achieve all optical packet switching and avoid $\mathrm{O} / \mathrm{E} / \mathrm{O}$ conversions that represent the bottleneck of the network.

With this kind of nodes one of the main issues is contention resolution in the optical domain, which arises when two or more packets contend for the same wavelength on the same fiber at the same time. Contention resolution can be potentially achieved in time, space, wavelength and code domain. Due to the lack of optical memories, most studies consider wavelength conversion as a way to solve contention. The main drawback of this approach is that tunable wavelength converters (TWCs) are very expensive components, in particular full range TWCs (FR-TWCs), that are able to convert each wavelength into each other. In fact when these converters are implemented by a single component, the output signal quality depends on the combination of input and output wavelengths. In particular when output wavelength is far from the input one, resulting output signal is significantly degradated [4]. For this reason, a FRTWC must be implemented by a cascade of more suitable wavelength converters with smaller conversion range, called limited range TWCs (LR-TWCs) [4]. The employment of LR-TWCs instead of FR-TWCs in a switch architecture leads to relevant cost saving.

To maintain switch cost low, switch architectures with limited number of shared tunable wavelength converters (TWCs), with full or limited conversion capability have been studied [4], [5]. Different architectures that employ TWCs shared on output links, referred as shared per link (SPL) [5], or shared among all input fibers and wavelengths, referred as shared per node (SPN), have been proposed in literature. It has been demonstrated that the same performance as fully equipped architecture can be obtained with different solutions by suitably calculating the number of TWCs, leading in some cases to relevant cost saving.

A buffer-less optical switching node equipped with shared per node TWCs in synchronous scenario is considered here. An example of this architecture is proposed in figure 1, where the switching node is equipped with $N$ input/output interfaces with one fiber carrying $M$ wavelengths. It can be seen that $R$ TWCs are grouped together in a single bank so that an incoming packet can exploit whatever TWC. Fully equipped architecture would require $N M$ TWCs. Here $R \leq N M$ TWCs are considered so packet loss can occur in the TWC bank. In each time slot input channels (wavelengths) are split and

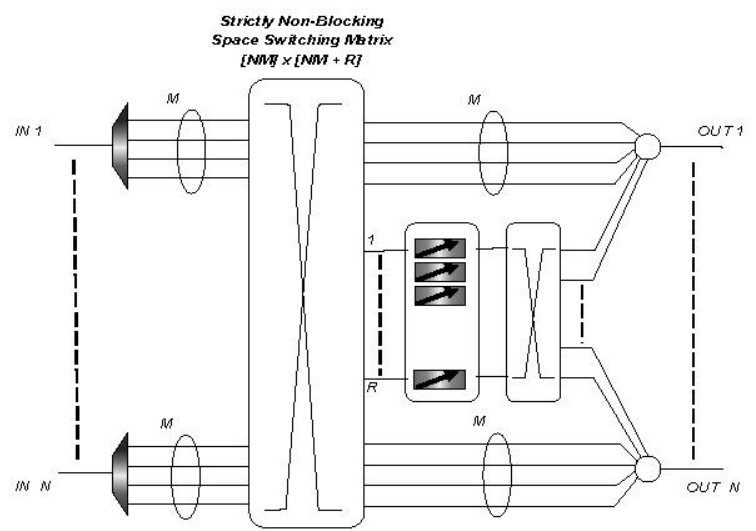

Fig. 1. Shared per node switching architecture with $N$ input and output fibers, $M$ wavelengths per fiber and $R$ TWCs.

incoming packets are synchronized. A first check is made to forward the incoming packets without wavelength conversion by exploiting strictly non-blocking space switch, otherwise the packet is sent to the TWC bank and forwarded, after wavelength conversion, if there is at least one available TWC. Channels on output interfaces are multiplexed by means of couplers. At the ingress of each coupler a maximum of $M$ packets, each carried by a different wavelength, is allowed. This architecture can be equipped with limited range TWCs 
(LR-TWCs), to assure better feasibility. An adequate number of LRTWCs is needed to provide performance similar to an architecture equipped with the maximum number $(N M)$ of full range wavelength converters (FR-TWCs). In switching node architectures equipped with LR-TWCs, a packet carried by wavelength $j$ can be converted in a sub-set of adjacent wavelengths next to $j$. This sub-set is named adjacency set $\left(A S_{j}\right)$ of wavelength $j$, and its cardinality is the conversion degree of wavelength $j$ [4]. In addition, in this paper circular symmetrical wavelength conversion is taken in account, that is a packet carried by wavelength $j$ can be converted in $d$ wavelengths on both sides of the wavelength $j$, and $d$ is called conversion range. The adjacency set of wavelength $j$ is defined as the interval $[(j-d+M)$ modulo $M,(j+d)$ modulo $M]$. With circular symmetrical wavelength conversion, all wavelengths have the same conversion degree, given by $2 d+1$. The difference between circular and non-circular wavelength conversion is presented in figure 2 , that illustrates the adjacency set of each wavelength in the system in case $M=8$ and $d=1$. It is possible to note that in case of noncircular symmetrical wavelength conversion, the adjacency set of the wavelengths near the boundaries have a smaller conversion degree than the wavelengths in the meddle.

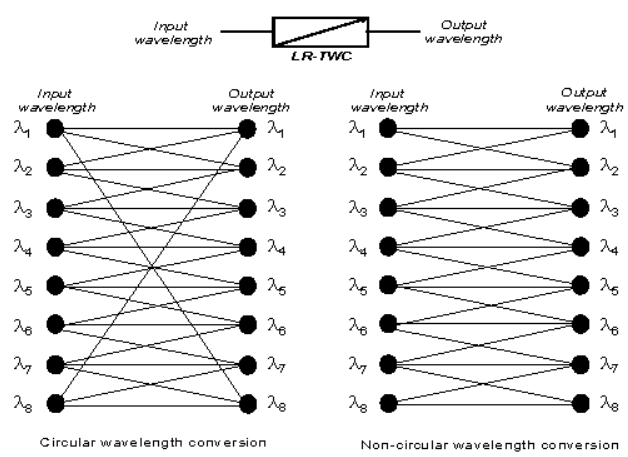

Fig. 2. Circular and non-circular wavelength conversion in case $M=8$ wavelengths per fiber and conversion range $d=1$.

The analytical representation of packet loss with limited range wavelength conversion is a non trivial problem; for this reason this paper considers circular symmetrical wavelength conversion only, which is simpler to represent. Very general analytical representation has been proposed in [4] to deal with a broader class of optical switches that comprises also this kind of architecture. Here a specific but very simple approach is proposed that is easy to be applied to tipical multistage switch implementation [6], [7]. First, a semianalytical model to evaluate packet loss probability is presented. In this case the contribution to the packet loss due to limited range wavelength conversion is evaluated by means of simulation, the other contributions are analytically evaluated. Then, a separate fully analytical approximate evaluation of the packet loss due to limited range wavelength conversion is provided to obtain an analytical expression of packet loss using discrete time Markov chains. Semianalytical model and the approximate expression are validated by means of simulation.

A time slot based scheduling algorithm similar to the one used in [5] is considered to manage packet forwarding. Other scheduling algorithms for this kind of switch, e.g. First Available Algorithm (FAA) and Optimal Scheduling Algorithm (OSA), can be found in [8]. The one applied here aims at maximizing the number of packets forwarded without wavelength conversion and was first proposed in [9].

The paper is organized as follows. In section II the semi-analytical model to evaluate packet loss in the SPN architecture equipped with LR-TWCs is described. Section III gives the approximate analytical expression of the packet loss due to limited range wavelength conversion. In section IV the model is validated by comparing analysis and simulation results and trade-off between performancecost effectiveness is demonstrated. Finally, in section V conclusions are carried out.

\section{SEMi-ANALytiCAL MODEL OF SPN SWitCH With LIMITED RANGE WAVELENGTH CONVERSION}

The proposed switching architecture can be used in different network contexts ranging from wavelength switching to optical packet/burst switching. Here the attention is focused on synchronous optical packet/burst switched networks with fixed-sized optical packets transferred through the network using a slotted statistical multiplexing scheme. Two main different traffic assumptions are considered regarding the arrivals on switch input channels:

- Bernoulli arrivals, meaning that arrivals in different slots are independent and characterized by the probability $p$ of an arrival in a slot

- admissible traffic, meaning that arrivals are still characterized by mean $p$ but no more than $M$ packets arrive in a slot for the same output fiber

Bernoulli traffic can be considered as representative of the traffic in connection-less optical packet/burst switched networks as the result of statistical multiplexing of an high number of packets generated by the edge assembly units [10]. The Bernoulli assumption is quite general but not far from reality. In fact it has been shown that the assembly process absorbs much of correlation existing in the incoming peripheral traffic, e.g. IP traffic [10]. admissible traffic could, on the other hand, be considered as the result of the admission operation performed on optical packets that makes the traffic at each node to avoid switch output overbooking in each time slot: no more than $M$ bursts are admitted on the same output fiber. Anyway, also admissible traffic needs wavelength conversion to resolve contention in the wavelength domain and could run into switch internal blocking due to switch resource unavailability. Fiber-to-fiber switching is considered meaning that a packet arriving on an input fiber $k$ and wavelength $j$ could in principle be forwarded to any output $l$ and wavelength $m$.

In the development of the analytical models a key hypothesis is that the maximum number of packets is forwarded without conversion. An incoming optical packet is forwarded without conversion if its wavelength is not in use on the requested output fiber, otherwise it is forwarded to the output fiber after wavelength conversion. With limited range wavelength converters (LR-TWCs), a packet carried by wavelength $j(j=1, \ldots, M)$ can be converted in one wavelength (randomly chosen) included on the adjacency set. The wavelengths that are far with respect the wavelength $j$, (outside from the adjacency set $A S_{j}$ ), are not available to forward the packet. The scheduling algorithm applied reflects this hypothesis.

An example of how the scheduling algorithm works is proposed in figure 3 . In the first step, packets carried by wavelength $j$ ( $j=$ $1, \ldots, M)$ and directed to output fiber $k(k=1, \ldots, N)$ are grouped (the corresponding group is called $L_{k}^{j}$ ). Packets in the same group contend for the same output channels, while packets on different groups are output contention free. In the second step one packet from each group (randomly chosen) is sent without conversion, so the maximum number of packets is forwarded without conversion. The other packets are sent by exploiting wavelength conversion, if possible (one of the packets of $L_{1}^{3}$ ). This packet can be forwarded because the free wavelength is included in the adjacency set of the wavelength the packet is carried $\left(A S_{3}\right)$. In fact the free wavelength is wavelength 4 , the packet is carried by wavelength 3 and the conversion range is $d=1$. Note that if there are more than one free wavelength in the adjacency set, the wavelength used is randomly chosen (this is the ipothesys made in the model). This algorithm is not the best scheduling algorithm when LR-TWCs are used as discussed 


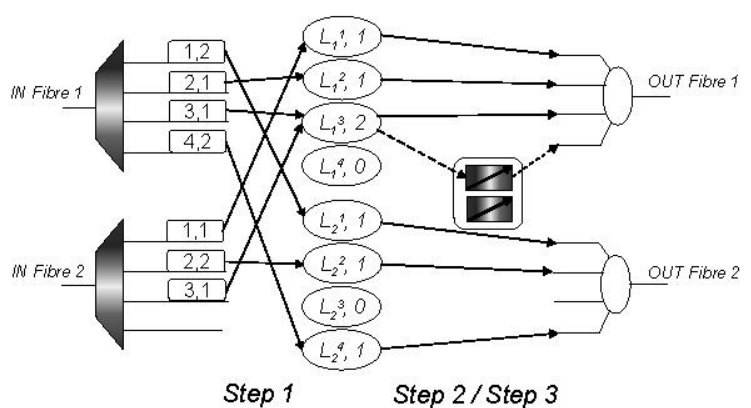

Step 1

Step 2 / Step 3

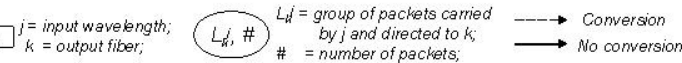

Fig. 3. Example of the scheduling algorithm in shared per node architecture with $N=2$ input/output fibers, $M=4$ wavelengths per fiber, $R=2$ LR-TWCs with conversion range $d=1$.

in [4], [8]. In these papers a better scheduling algorithm, called FAA (first available algorithm) is presented, in order to minimize the packet loss probability with light load. With this algorithm, some packets can be converted even if its wavelength is not in use, in order to improve the reachability of the wavelengths, that is limited due to the limited range. Anyway, with FAA algorithm, packet loss is minimized with light load, but when the load is high, this algorithm leads to a higher packet loss. In addition, in this algorithm the number of packets forwarded without conversion is not maximized, and this differs from the hypothesis made in the development of the models presented in this paper. The semi-analytical representation of the packet loss is described in the following for Bernoulli and admissible traffics.

Bernoulli traffic. Bernoulli arrivals are assumed with probability $p$ on each wavelength in a time slot. Arrivals on different input wavelengths are independent and are addressed to the output fibers with the same probability $1 / N$.

In the proposed model the packet loss probability is evaluated following a tagged incoming packet carried by wavelength $j$ and directed to output fiber $k$. Packet loss occurs if one of the following events occurs:

- loss due to output contention: the packet loses contention on output fiber because excess packets require channels on that fiber in the same time slot; the probability of this event is indicated with $P_{u}$

- loss due to limited range: the packet is not blocked on output fiber, it requires conversion but it loses contention on its adjacency set because excess packets (more than $2 d$ ) require conversion in the same adjacency set in the same time slot; the probability of this event is indicated with $P_{l r}$

- loss due to limited number of LR-TWCs: the packet requires conversion and it is not blocked due to limited range, but loses contention on wavelength converters because excess packets (more than $R$ ) require to exploit LR-TWCs in the same time slot; the probability of this event is indicated with $P_{\text {bwc }}$

In figure 4 an example of packet loss due to the three different events is presented, in case $N=2, M=4, R=2$ and $d=1$. In 4(a) a packet in group $L_{1}^{2}$ is lost due to output contention, because no more output wavelength channels are available. Instead in 4(b) a packet in group $L_{1}^{1}$ is lost due to limited range of LR-TWCs. In fact in this case the free output wavelength is out of the adjacency set $\left(A S_{1}\right)$ of the wavelength the packet is carried (1). Finally, in 4(c) a packet in group $L_{2}^{4}$ is lost due to the lack of LR-TWCs. Note that in this case there is a free wavelength in the adjacency set, but there are not enough LR-TWCs to satisfy all conversion requests.

The second and third events take into account limitations in conversion capability, so this two terms can be viewed as related

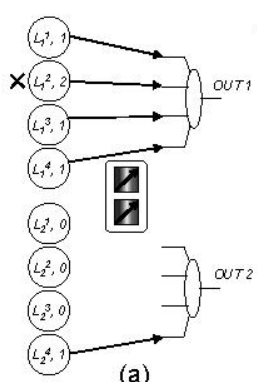

(a)

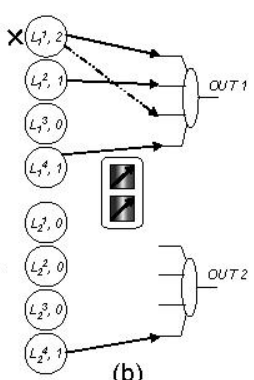

(b)

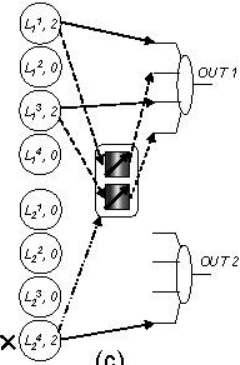

(c)
Fig. 4. Example of packet loss due to: (a) output contention, (b) limited conversion range, (c) lack of LR-TWCs

to inconvertibility of the tagged packet. Packet loss due to inconvertibility $P_{\text {inconv }}$ is defined as:

$$
P_{\text {inconv }}=P_{i r}+\left(1-P_{i r}\right) P_{b w c}
$$

The first term $P_{l r}$ is the packet loss due to limited range, the second one is the joint probability that the packet is not lost due to limited range $\left(1-P_{l r}\right)$ and the probability that the packet is lost due to lack of LR-TWCs $\left(P_{b w c}\right)$.

Moreover, a packet must be converted when it is blocked on its wavelength in the destination output fiber, and the probability of this event is indicated with $P_{b}$. The expression of the overall packet loss probability that takes in account the three above contributions is:

$$
P_{\text {loss }}=P_{u}+P_{b}\left(1-\frac{P_{u}}{P_{b}}\right) P_{\text {inconv }}
$$

The first term $P_{u}$ is the probability that a packet is lost because of output blocking. The second term is the joint probability of $P_{b}\left(1-\frac{P_{u}}{P_{b}}\right)$ and $P_{\text {inconv. The former represents the probability }}$ that the tagged packet is blocked on its wavelength $\left(P_{b}\right)$ and it is not blocked on output $\left(1-\frac{P_{u}}{P_{u}}\right)$, then the probability that the packet requires conversion. The latter is the packet loss probability due to inconvertibility of the tagged packet. The overall packet loss probability can also be written as:

$$
P_{\text {loss }}=P_{u}+P_{b}\left(1-\frac{P_{u}}{P_{b}}\right)\left(P_{l r}+\left(1-P_{l r}\right) P_{b w c}\right)
$$

$P_{u}, P_{b}, P_{b w c}$ are evaluated by means of analytical expressions, while $P_{i r}$ is evaluated by means of simulation. The probability $P_{u}$ that the tagged packet is blocked on the destination output fiber is:

$$
P_{u}=\sum_{h=M+1}^{N M}\left(1-\frac{M}{h}\right)\left(\begin{array}{c}
N M-1 \\
h-1
\end{array}\right)\left(\frac{p}{N}\right)^{h-1}\left(1-\frac{p}{N}\right)^{N M-h}
$$

where the probability of $h$ arrivals directed to destination output fiber is expressed as the probability of $h-1$ arrivals more than the tagged from the other $N M-1$ input channels. There are up to $N M$ arrivals directed to target output fiber and only $M$ can be transmitted on output wavelengths. Loss occurs when there are more than $M$ arrivals and the tagged packet is not one of those chosen for transmission on output channels (wavelengths).

The probability $P_{b}$ that the tagged packet is not forwarded into its wavelengths is given by:

$$
P_{b}=\sum_{h=2}^{N}\left(1-\frac{1}{h}\right)\left(\begin{array}{c}
N-1 \\
h-1
\end{array}\right)\left(\frac{p}{N}\right)^{h-1}\left(1-\frac{p}{N}\right)^{N-h}
$$

by considering that there are $N$ input fibers and the wavelengths are replicated in each of them, it is possible to have up to $N$ packet 
arrivals directed to the same output fiber and carried by the same wavelength.

As before mentioned, the probability that the tagged packet requires conversion is given by $1-\frac{P_{u}}{P_{b}}$. The tagged packet is sent to the LR-TWC bank if and only if it requires conversion and it is not lost due to the limited range, consequently the average load offered to the LR-TWC bank by a single wavelength is:

$$
A_{w c}=p P_{b}\left(1-\frac{P_{u}}{P_{b}}\right)\left(1-P_{l r}\right)
$$

where the probability that the packet is lost due to limited range is also taken into account. Packet loss probability in the LR-TWCs bank occurs when there are more than $R$ requests to access LR-TWCs. The assumption of $N M$ independent Bernoulli arrivals on the LR-TWC bank in a time slot is made. As a matter of fact the arrivals on the LRTWC bank in a given time slot are not independent and are negatively correlated since, for a switch with $N$ input/output fibers, the total number of new packets arriving each time slot in the same wavelength is no greater than $N$. As a consequence each packet addressed to the output fiber $g$ reduces the likelihood of packets destined for output fiber $k$, for $g \neq k$. In the extreme case, if $N$ packets arrive during a time slot for a single output fiber $g$, no packet can arrive for any of the other output fibers [11]. In [11] it is shown that the effects of this correlation are sensible only when the load per wavelength is high, they can be neglected otherwise. In this context the correlation can be omitted, because, when the load is high, the packet loss due to the lack of LR-TWCs is shadowed by the contention on output fiber (as will be proved in section IV). Further, the effect of this negative correlation decreases when the switching size $N$ increases. Under this hypothesis, the packet loss probability due to the lack of LR-TWCs, $P_{b w c}$, is calculated as:

$P_{b w c}=\sum_{h=R+1}^{N M}\left(1-\frac{R}{h}\right)\left(\begin{array}{c}N M-1 \\ h-1\end{array}\right)\left(A_{w c}\right)^{h-1}\left(1-A_{w c}\right)^{N M-h}$

When $R=N M$ (fully equipped architecture), $P_{b w c}=0, P_{\text {inconv }}=$ $P_{l_{r}}$ and $P_{\text {loss }}=P_{u}+P_{b}\left(1-\frac{P_{u}}{P_{b}}\right) P_{l r}$. Instead when $R=0, P_{b w c}=1$ and $P_{\text {loss }}=P_{b}$, in fact if one packet is blocked on its wavelength, it is lost because no conversion is possible. This model allows to find the minimum number of LR-TWCs that leads to similar packet loss as full wavelength conversion case, limiting switch cost.

Packet loss probability $P_{l r}$ is evaluated by means of simulation and used to evaluate the packet loss due to the lack of LR-TWCs, so that the overall expression of the packet loss can be obtained by applying formula (3).

Note that the expression of the packet loss takes into account the different contributions to the packet loss. If different hypothesis are made in the packet forwarding it is necessary to change only the expression of some contributions while the overall expression of $P_{\text {loss }}$ is maintained.

admissible traffic. When admissible traffic is considered, no more than $M$ packets addressed to the same output port arrive in a time slot. In this situation, the architecture is output contention free, so $P_{u}=$ 0 . So no more than $M$ packets can arrive on the same wavelength addressed to the same output fiber, given that for the same output fiber there are maximum $M$ packet arrivals in total. The traffic offered to the LR-TWCs by a single output wavelength is evaluated by taking into account the constraint of maximum $M$ packets addressed to the tagged output fiber. The expression of $A_{w c}$ results in:

$$
A_{w c}=p P_{b}\left(1-P_{l r}\right)
$$

By considering independent arrivals at the LR-TWC bank, $P_{b w c}$ can be calculated using (7) and the final expression of the packet loss with admissible traffic is:

$$
P_{\text {loss }}=P_{b}\left(P_{l r}+\left(1-P_{l r}\right) P_{b w c}\right)
$$

where $P_{b}$ is given by:

$$
P_{b}=\frac{\sum_{h=2}^{\min \{N, M\}}\left(1-\frac{1}{h}\right)\left(\begin{array}{c}
N-1 \\
h-1
\end{array}\right)\left(\frac{p}{N}\right)^{h-1}\left(1-\frac{p}{N}\right)^{N-h}}{\sum_{h=2}^{\min \{N, M\}}\left(\begin{array}{c}
N \\
h
\end{array}\right)\left(\frac{p}{N}\right)^{h}\left(1-\frac{p}{N}\right)^{N-h}}
$$

In this case the independence assumption is less accurate than for Bernoulli traffic, due to the finite set of arrivals according to the admission procedure that enhance correlations as will be shown in model validation.

\section{APPROXIMATE EXPRESSION OF PACKET LOSS \\ PROBABILITY DUE TO LIMITED RANGE WAVELENGTH CONVERSION}

In this section, an approximate analytical expression for the packet loss due to limited range is presented for Bernoulli traffic assumption. A discrete time analytical model is proposed to evaluate the performance with limited range wavelength converters, which is based on discrete-time Markov chains. A similar approach was proposed in [12] for shared per link wavelength converters. For this purpose, we construct a two-dimensional Markov chain with the state space consisting of pairs $(i, j), i=1, \ldots, N M$ and $j=1, \ldots, M$. There are $K=N M$ input wavelength channels and $i$ keeps track of the input channels. The $j$ component, on the other hand, keeps track of the number of occupied channels at a designated output fiber. Note that, there is a packet arrival with probability $p$ on each input wavelength channel. The Markov chain evolves as follows; when the system is at state $(i, j), i<K$ with a probability $p$ there is a new optical packet. In this case, this optical packet is directed to the designated output fiber with probability $1 / N$. When this happens, with a probability of $j / M$, the packet will require conversion and with probability $1-j / M$, the packet will be admitted onto the fiber. When conversion is required, with a probability of $P_{l_{r}}(j)$, the tuning range will be occupied and the packet will be dropped. Here, $P_{l r}(j)$ denotes the loss probability due to limited range when there are $j$ occupied channels at the designated fiber and can be approximated by the following expression (also see [12]):

$$
P_{l r}(j)= \begin{cases}\frac{\left(\begin{array}{c}
M-d-1 \\
j-1-d
\end{array}\right)}{\left(\begin{array}{c}
M-1 \\
j-1
\end{array}\right)}=\frac{j-1}{M-1} \frac{j-2}{M-2} \cdots \frac{j-d}{M-d} & \text { if } j \geq d+1, \\
0 & \text { if } j \leq d .\end{cases}
$$

On the other hand, with a probability of $1-P_{l r}(j)$, there is at least one idle channel in the tuning range and the optical packet will be forwarded to the converter bank at which it will face a blocking probability $P_{b w c}$. Let $P_{(i, j),(k, l)}$ denote the state transition probability from a given state $(i, j)$ to $(k, l)$. For a given $P_{b w c}$,

$$
\left\{\begin{array}{l}
P_{(i, j),(i+1, j+1)}=\frac{1}{N M}\left(p(M-j)+p j\left(1-P_{l r}(j)\right)\left(1-P_{b w c}\right)\right) \\
i<K, j<M \\
P_{(i, j),(i+1, j)}=1-P_{(i, j),(i+1, j+1)}, i<K, j<M \\
P_{(i, M),(i+1, M)}=1, i<K \\
P_{(K, j),(1,0)}=1-p / N, \forall j \\
P_{(K, j),(1,1)}=p / N \cdot \forall j
\end{array}\right.
$$

Now let $\pi(i, j)$ denote the steady-state probability of being in state $(i, j)$. The probability that a certain input channel to have a packet directed to the converter pool is written as

$$
P_{c p}=\sum_{i, j<M} \pi(i, j) p j\left(1-P_{l r}(j)\right) / M
$$


and the above equation then gives us an expression for $P_{b w c}$ :

$$
P_{b w c}=\sum_{k=R+1}^{K}\left(\begin{array}{l}
K \\
k
\end{array}\right) P_{c p}^{k}\left(1-P_{c p}\right)^{K-k} .
$$

To summarize, we start with a fixed $P_{b w c}$ and obtain a new expression for $P_{b w c}$ in (14) which is a fixed point relationship. We propose in this paper to use a fixed point iteration to find $P_{b w c}$ and consequently we write the loss probability $P_{\text {loss }}=$

$$
\sum_{i, j<M} j \pi(i, j) / M\left(P_{l r}(j)+\left(1-P_{l r}(j)\right) P_{b w c}\right)+\sum_{i, j=M} \pi(i, j)
$$

\section{Numerical RESUlts}

In this section analytical and simulation results are compared. Simulation results have been obtained by applying the scheduling algorithm described in II and considering a confidence interval at $95 \%$ less than or equal to $5 \%$ of the mean.

First, in figure 5 a comparison between packet loss probability obtained with semi-analytical model and simulation under Bernoulli traffic is shown. This figure plots the packet loss probability as a function of the number $R$ of LR-TWCs, varying the conversion range $(d=1,2,3)$, in case $N=16, M=8, p=0.3,0.7$. It is possible to see very good agreement between simulation and semi-analytical results, that exploit the value of $P_{l_{r}}$ evaluated by means of simulation. In fact, a simulator that is able to evaluate the different contributions to packet loss has been developed. This simulator is very helpful to understand the entity of the approximation introduced in the analytical model. In figure 6 the various packet loss contributions

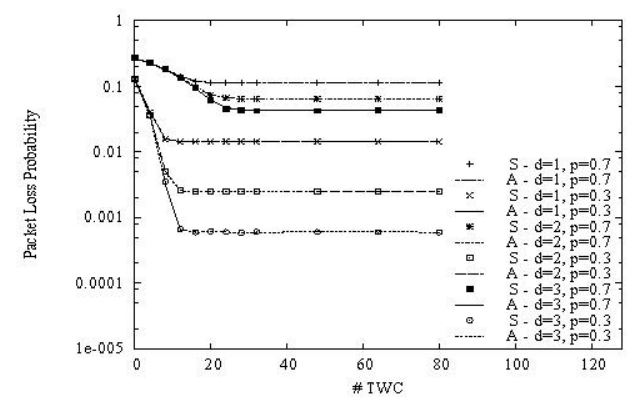

Fig. 5. Packet loss probability with Bernoulli traffic as a function of the number $R$ of LR-TWCs obtained with semi-analytical and simulation approaches, varying the conversion range $(d=1,2,3)$ in case $N=16$, $M=8, p=0.3,0.7$

obtained by simulation and semi-analytical model for Bernoulli traffic are illustrated and compared, in case $N=16, M=8, p=0.7$ and $d=3$. Contributions and compared using simulation results and the semi-analytical model, in case $N=16, M=8, p=0.7$ and $d=3$. It is possible to see perfect agreement between the values of $P_{u b}$ and $P_{b}$ evaluated using analytical and simulation approach, while $P_{b w c}$ evaluated by means of analysis slightly overcomes the simulated one when $R$ increases, due to the independent arrivals hypothesis, as explained in II. Anyway, this difference is evident when $P_{b w c}$ is very low, so that the total packet loss shows very good agreement between semi-analytical and simulation results. In figure 7 the comparison between packet loss probability with Bernoulli traffic as a function of the conversion range $d$ in case $N=16, M=32, p=0.7$ varying the number of LR-TWCs available is shown. A very good agreement between semi-analytical and simulation results is present. The little difference for $R=96$ is due to the approximated evaluation of $P_{b w c}$ introduced by the model, as already discussed for figure 6 .

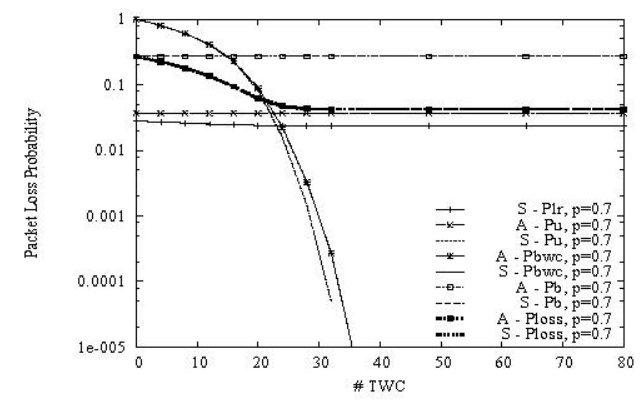

Fig. 6. Packet loss probabilities $P_{b}, P_{u}, P_{b w c}, P_{\text {loss }}$ with Bernoulli traffic as a function of the number $R$ of LR-TWCs evaluated with semi-analytical and simulation approaches, in case $N=16, M=8, p=0.7$ and $d=3$

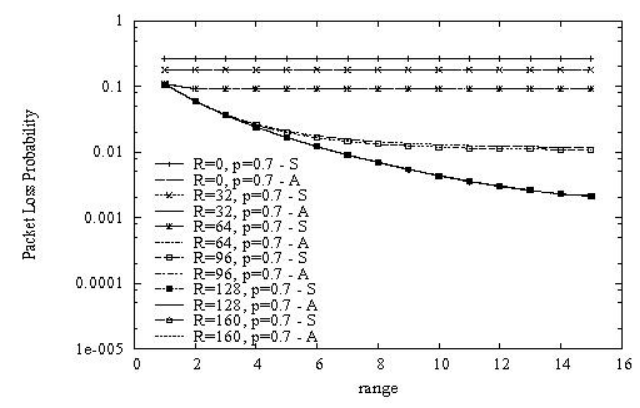

Fig. 7. Packet loss probability with Bernoulli traffic as a function of the conversion range $d$, varying the number $R$ of LR-TWCs available, in case $N=16, M=32$ and $p=0.7$

In figure 8 results obtained with the analytical approach illustrated in section III are compared with simulation in case $N=16, M=8$ and $p=0.3$. The figure presents the packet loss as a function of the number $R$ of LR-TWCs, varying the conversion range $d$. In this case it is possible to see that the model slightly underestimates the packet loss obtained by simulation. Analogous results are now provided with

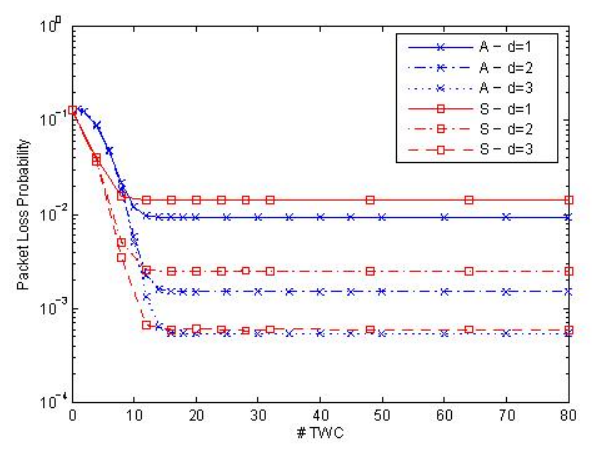

Fig. 8. Packet loss probability with Bernoulli traffic as a function of the number $R$ of LR-TWCS, varying the conversion range $d$, in case $N=16$, $M=8$ and $p=0.3$. Simulation (S) and analytical (A) results are compared

admissible traffic. In figure 9 a comparison between $P_{b}, P_{b w c}$ and $P_{\text {loss }}$ obtained by means of simulation and semi-analytical approach is presented in case $N=16, M=8, p=0.7$ and $d=3$. In this case the independence arrivals hypothesis made in the evaluation of $P_{\text {bwc }}$ leads to overcome the value obtained by simulation, so that there is a little difference in the packet loss probability $P_{\text {loss. }}$. Finally 


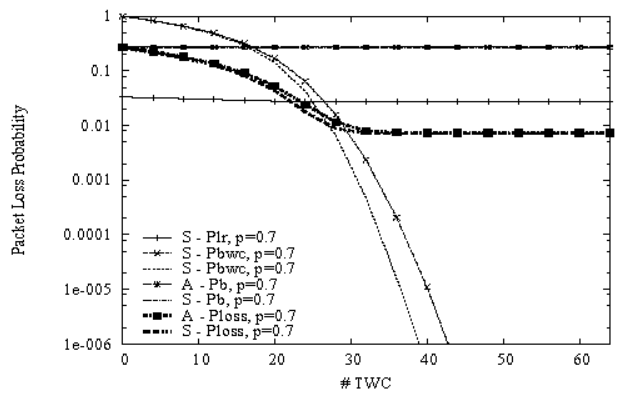

Fig. 9. Packet loss probabilities $P_{b}, P_{u}, P_{b w c}, P_{l o s s}$ with admissible traffic as a function of the number $R$ of LR-TWCs obtained with semi-analytical and simulation approaches, in case $N=16, M=8, p=0.7$ and $d=3$

in figures 10 and 11 the packet loss probability with admissible traffic as a function of the number $R$ of LR-TWCs varying load is illustrated, in case $N=16, M=8$ and $d=3(10), d=4$ (11). When $d=4$ TWCs are full range (FR-TWCs), packet loss due to limited range is $P_{l r}=0$ and in this case the packet loss probability with admissible traffic tends to 0 when the number of FR-TWCs $R$ increases, due to the absence of output contention. When the range is limited, $d=3$, this is not possible due to the presence of the packet loss due to limited range, $P_{l r}$ that represents the asymptotic value of the packet loss. In the former case ( $d=4$, full range TWCs), when the load is high, the analytical expression of packet loss overcomes the simulated, in the latter the difference is much less evident due to the presence of $P_{l r}$.

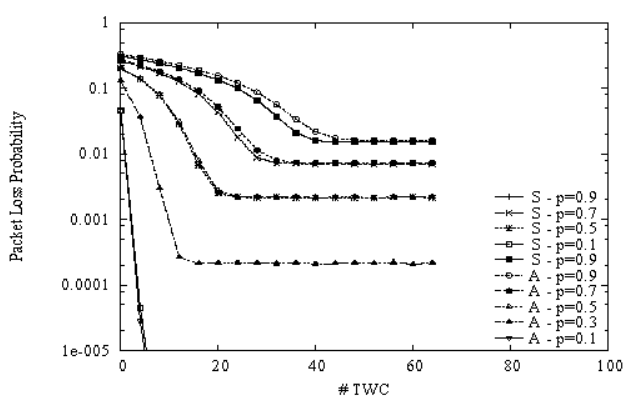

Fig. 10. Packet loss probability with admissible traffic as a function of the number $R$ of LR-TWCs, varying load $p$ in case $N=16, M=8, d=3$

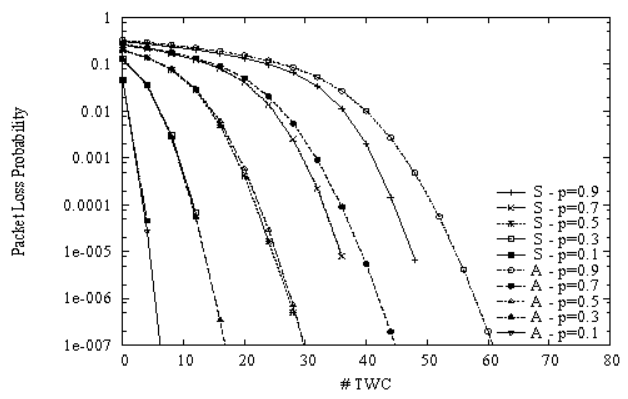

Fig. 11. Packet loss probability with admissible traffic as a function of the number $R$ of LR-TWCs, varying load $p$ in case $N=16, M=8, d=4$

\section{CONCLUSIONS}

In this paper a synchronous buffer-less switch with limited range shared per node wavelength converters has been evaluated. An analytical framework that takes into account the different contributions to packet loss is defined and is shown to lead to significant insight of switch subsystems behavior. Semi-analytical results show that the approach is accurate by first providing the evaluation of loss due to limited range wavelength conversion constraint through simulation, both with Bernoulli and admissible traffic. A fully analytical approximate method for Bernoulli traffic has been also set up that captures the main loss behavior but requires some refinements to fit well with simulation results. The open point is the accurate evaluation of the packet loss rate $P_{l_{r}}$ due to wavelength conversion range limitation which is left for further work

\section{ACKNOWLEDGMENTS}

This work was partially funded by the Italian Ministry of Education, University and Research through the OSATE project 2005095981 and by the Commission of European Union through the IST-FP6 Network of Excellence e-Photon/ONe. The authors wishes also to thank the anonymous reviewers for the useful suggestions to improve the paper.

\section{REFERENCES}

[1] M. J. O'Mahony, D. Simeonidou, D. K. Hunter, A. Tzanakaki, "The application of Optical Packet Switching in Future Communications Networks", IEEE Communication Magazine, vol. 39, no. 3, pp.128-135, Mar. 2001.

[2] J. Xu, H. Perros, G. Rouskas, "Techniques for Optical Packet Switching and Optical Burst Switching", IEEE Communication Magazine, Vol. 39, no. 1 , pp. 136-142, January 2001.

[3] B. Li, Y. Qin, X. Cao, K. Sivalingam, "Photonic Packet Switching: Architectures and Performance", Opt. Network Magazine, pp. 27-39, $\mathrm{Jan} / \mathrm{Feb} .2001$

[4] V. Eramo, M. Listanti, M. Spaziani, "Resource Sharing in Optical Packet Switches With Limited-Range Wavelength Converters", Journal of Lightwave Technology, Vol.23, No. 2, Feb. 2005.

[5] V. Eramo, M. Listanti, P. Pacifici, "A Comparison Study on the Number of Wavelength Converters Needed in Synchronous and Asynchronous All-Optical Switching Architectures", Joumal of Lightwave Technology, Vol.21, No. 2, Feb. 2003

[6] C. Raffaelli, M. Savi, A. Stavdas, "Sharing Wavelength Converters in Multistage Optical Packet Switches", IEEE Workshop on High Performance Switching and Routing, HPSR, Poznan, Poland, 7-9 June 2006.

[7] C. Raffaelli, M. Savi, A. Stavdas "Performance of Scheduling Algorithms in Multi-Stage Optical Packet Switches with Sparse Wavelength Converters", Proceedings of IEEE Globecom 2006, San Francisco, U.S.A., 27 November-1 December 2006.

[8] V. Eramo, M. Listanti, "Performance of scheduling algorithms minimizing the packet loss in optical switches equipped with limited range and shared wavelength converters", Broadband Networks, 2005 2nd International Conference on, 3-7 Oct. 2005, Page(s):1096 - 1105 Vol. 2 .

[9] V. Eramo, M. Listanti "Performance Evaluation of a Bufferless Optical Packet Switch With Limited-Range Wavelength Converters", IEEE Photonics Technology Letters, Vol. 16, No. 2, February 2004.

[10] M. Izal, J. Aracil, "On the Influence of Self Similarity on Optical Burst Switching Traffic", Proceedings of Globecom 2002.

[11] A. E. Eckberg, T.-C. Hou "Effects of output buffer sharing on buffer requirements in an ATDM packet switching", INFOCOM '88. Networks: Evolution or Revolution? Proceedings. Seventh Annual Joint Conference of the IEEE Computer and Communcations Societies., 27-31 March 1988 Page(s): 459 - 466.

[12] K. Dogan, N. Akar,"A Performance Study of Limited Range Partial Wavelength Conversion for Asynchronous Optical Packet/Burst Switching"', Proceedings ICC 2006, Istanbul, Turkey. 